\title{
Automated detection of a preseizure state based on a decrease in synchronization in intracranial electroencephalogram recordings from epilepsy patients
}

\author{
Florian Mormann, ${ }^{1,2, *}$ Ralph G. Andrzejak, ${ }^{3}$ Thomas Kreuz, ${ }^{1,3}$ Christoph Rieke,,${ }^{1,2}$ Peter David, ${ }^{2}$ Christian E. Elger, ${ }^{1}$ \\ and Klaus Lehnertz ${ }^{1}$ \\ ${ }^{1}$ Department of Epileptology, University of Bonn, Sigmund-Freud-Straße 25, 53105 Bonn, Germany \\ ${ }^{2}$ Helmholtz-Institut für Strahlen- und Kernphysik, University of Bonn, Nußallee 14-16, 53115 Bonn, Germany \\ ${ }^{3}$ John-von-Neumann Institute for Computing, Forschungszentrum Jülich, 52425 Jülich, Germany
}

(Received 30 August 2002; published 26 February 2003)

\begin{abstract}
The question whether information extracted from the electroencephalogram (EEG) of epilepsy patients can be used for the prediction of seizures has recently attracted much attention. Several studies have reported evidence for the existence of a preseizure state that can be detected using different measures derived from the theory of dynamical systems. Most of these studies, however, have neglected to sufficiently investigate the specificity of the observed effects or suffer from other methodological shortcomings. In this paper we present an automated technique for the detection of a preseizure state from EEG recordings using two different measures for synchronization between recording sites, namely, the mean phase coherence as a measure for phase synchronization and the maximum linear cross correlation as a measure for lag synchronization. Based on the observation of characteristic drops in synchronization prior to seizure onset, we used this phenomenon for the characterization of a preseizure state and its distinction from the remaining seizure-free interval. After optimizing our technique on a group of 10 patients with temporal lobe epilepsy we obtained a successful detection of a preseizure state prior to 12 out of 14 analyzed seizures for both measures at a very high specificity as tested on recordings from the seizure-free interval. After checking for in-sample overtraining via cross validation, we applied a surrogate test to validate the observed predictability. Based on our results, we discuss the differences of the two synchronization measures in terms of the dynamics underlying seizure generation in focal epilepsies.
\end{abstract}

DOI: 10.1103/PhysRevE.67.021912 PACS number(s): 87.19.La, 05.45.Xt, 05.45.Tp, 05.10.-a

\section{INTRODUCTION}

An important issue in epileptology is the question whether epileptic seizures can be anticipated prior to their occurrence. Much research has been done on this topic, and recent studies have shown that a number of characterizing measures derived from the theory of dynamical systems are to some extent capable of extracting information from the electroencephalogram (EEG) that allow the definition of a preictal state, i.e., the state preceding a seizure (ictus).

After some early work on the predictability of seizures dating back to the 1970s [1], attempts to extract seizure precursors from the EEG were carried out by different groups using mostly linear approaches $[2,3]$ or analysis of spike occurrence [4-7]. The earliest attempts to use nonlinear time series analysis were started in the 1990s [8,9] using the largest Lyapunov exponent to describe changes in brain dynamics. The first studies to describe characteristic changes shortly before an impending seizure in a larger group of patients [10-13] used the correlation dimension as a measure for neuronal complexity in the EEG or the correlation density, respectively. These studies were followed by others using measures such as dynamic similarity [14-17]. In a recent study, certain signal patterns ("bursts") and changes in signal energy [18] were reported to be of predictive value. Common to all of these analyses is the fact that they employ univariate measures. It is only recently that bivariate mea-

*Electronic address: fmormann@yahoo.de sures, namely, the difference between the largest Lyapunov exponents of two channels [19], and nonlinear interdependence measures [20], as well a multivariate approach based on simulated neuronal cell models [21], have been applied to the EEG of epilepsy patients.

A major problem with most of the studies presented to date is that they do not sufficiently (or not at all) investigate the specificity of the described precursors by analyzing interictal EEGs (i.e., EEGs recorded during the seizure-free interval) as control. Furthermore, many of these studies rely on $a$ posteriori knowledge, e.g., by selecting the best channel out of a large number of channels, or bear the risk of an insample overtraining of parameters used to calculate measures for the extraction of predictive information.

In our earlier work [22], we analyzed the degree of phase synchronization between EEG signals from different recording sites and found a sensitivity to both physiological and pathological synchronization in patients with temporal lobe epilepsy. In particular, we discovered the phenomenon of a distinct drop in synchronization before seizures that was usually not found during the interictal state. This decrease in synchronization was found to occur well in advance, sometimes hours before a seizure, leading us to conclude that a seizure may be seen as the mere "tip of the iceberg" [54] in the sense of it being the climax of successive changes in brain dynamics that start long before the actual seizure. These findings have since then been confirmed by another study [23] qualitatively describing preictal drops in phase synchronization in patients with focal epilepsies of neocortical origin. 
In the present study, we characterize the degree of synchronization between EEG signals recorded continuously from different locations within the brain and retrospectively analyze changes over time using the mean phase coherence as a measure for phase synchronization and the maximum linear cross correlation as a measure for lag synchronization. Our specific aim is to design an automated technique for the detection of a preictal state and to test its performance in terms of both sensitivity and specificity on a group of ten patients with temporal lobe epilepsy. The parameters of the underlying algorithm are optimized for the entire set of patients and the apparent performance is checked for in-sample overtraining via cross validation. A surrogate test for the entire set of recordings is carried out to serve as a test for statistical validity. Finally, the results obtained using the two different synchronization measures are compared and discussed.

\section{MATERIALS AND METHODS}

\section{A. Phase synchronization and mean phase coherence}

One of the types of synchronization examined in this study, the so-called phase synchronization, is the oldest concept of synchronization in physics [24] and was originally introduced for the description of two coupled harmonic (linear) oscillators [25]. Only recently has this concept been extended to nonlinear time series [26] and applied to biological time series such as the electrocardiogram and respiratory signals from healthy humans [27-30] as well as magnetoencephalograms and electromyograms from signals from Parkinsonian patients [31] and EEG signals from both epilepsy patients [22] and animal models of epilepsy [32]. Traditionally, phase synchronization is defined as the locking of the phases of two oscillating systems $a$ and $b$ :

$$
\phi_{a}(t)-\phi_{b}(t)=\text { const. }
$$

To quantify the degree of phase synchronization for two time series $s_{a}$ and $s_{b}$ we use the mean phase coherence defined as

$$
R=\left|\frac{1}{N} \sum_{j=0}^{N-1} e^{i\left[\phi_{a}(j \Delta t)-\phi_{b}(j \Delta t)\right]}\right|=1-V_{C}
$$

where $1 / \Delta t$ is the sampling rate of the discrete time series of length $N$ and $V_{C}$ denotes the circular variance [33] of an angular distribution obtained by transforming the differences in phase onto the unit circle in the complex plane. (In the literature, $R$ is sometimes also referred to as the intensity of the first Fourier mode of the phase distribution [34].) By definition $R$ is restricted to the interval $[0,1]$ and reaches the value 1 if and only if the condition of phase locking is obeyed, whereas a uniform distribution of phases (which would be expected, on the average, for unsynchronized time series) will result in $R=0$.

Note that by definition the mean phase coherence is different from other statistical measures for phase synchronization described in the literature such as the index based on conditional probability and the index based on Shannon entropy [31] but that all of them reflect the same properties of the dynamics. Nevertheless, all analyses in this study were carried out for the latter two measures as well. Since results were almost identical for all three measures, in the following we only report on results obtained using $R$ as a measure for phase synchronization.

In order to determine the mean phase coherence of two signals $s_{a}(t)$ and $s_{b}(t)$, it is first of all necessary to determine their phases $\phi_{a}(t)$ and $\phi_{b}(t)$. For this purpose we follow the analytic signal approach $[35,36]$ which renders an unambiguous definition of the so-called instantaneous phase for an arbitrary signal $s(t)$ :

$$
\phi(t)=\arctan \frac{\tilde{s}(t)}{s(t)},
$$

where

$$
\tilde{s}(t)=\frac{1}{\pi} \mathrm{P} \int_{-\infty}^{+\infty} \frac{s\left(t^{\prime}\right)}{t-t^{\prime}} d t^{\prime}
$$

is the Hilbert transform of the signal ( $\mathrm{P}$ denoting the Cauchy principal value). Application of the convolution theorem turns the last equation into

$$
\tilde{s}(t)=-i \cdot \mathcal{F}^{-1}\{\mathcal{F}[s(t)] \operatorname{sign}(\omega)\},
$$

where $\mathcal{F}$ denotes the Fourier transform and $\mathcal{F}^{-1}$ the inverse Fourier transform. From this notation it becomes evident that the Hilbert transform performs a phase shift of the original signal by $\pi / 2$ in the frequency domain while the power spectrum remains unchanged. Note that another way of defining the phase variable based on the wavelet transform has been proposed [37]. Recently, it has been shown, however, that this definition is closely related to the definition based on the Hilbert transform [32].

\section{B. Lag synchronization and maximum linear cross correlation}

A further type of synchronization is the lag synchronization [38] which is characterized by a condition in which the state variables $s_{a}(t)$ and $s_{b}(t)$ of two systems are shifted by a time lag $\tau$ but are otherwise identical:

$$
s_{a}(t+\tau)=s_{b}(t)
$$

A common measure for the similarity of two signals $s_{a}(t)$ and $s_{b}(t)$ is the linear cross correlation function defined as

$$
\operatorname{corr}\left(s_{a}, s_{b}\right)(\tau)=\int_{-\infty}^{+\infty} s_{a}(t+\tau) s_{b}(t) d t
$$

This function yields high values for such time lags $\tau$ for which the signals $s_{a}(t)$ and $s_{b}(t+\tau)$ have a similar course in time. It is therefore well suited as a quantitative measure for lag synchronization. In order to keep this measure independent of the variance of the signals $s_{a}(t)$ and $s_{b}(t)$, we use a normalized cross correlation function 


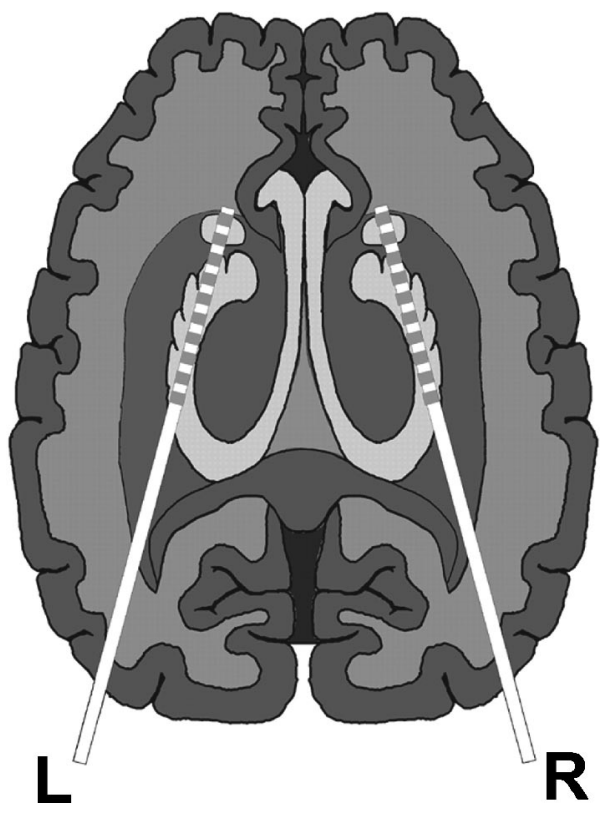

FIG. 1. Schematic view of intrahippocampal depth electrodes.

$$
C\left(s_{a}, s_{b}\right)(\tau)=\left|\frac{\operatorname{corr}\left(s_{a}, s_{b}\right)(\tau)}{\sqrt{\operatorname{corr}\left(s_{a}, s_{a}\right)(0) \cdot \operatorname{corr}\left(s_{b}, s_{b}\right)(0)}}\right|,
$$

where $\operatorname{corr}\left(s_{a}, s_{a}\right)(0)$ and $\operatorname{corr}\left(s_{b}, s_{b}\right)(0)$ are the values of the linear autocorrelation functions at $\tau=0$ limiting the range of the cross correlation function to the interval $[0,1]$. As an actual measure for the degree of lag synchronization we use the maximum linear cross correlation

$$
C_{\max }=\max _{\tau}\left\{C\left(s_{a}, s_{b}\right)(\tau)\right\}
$$

where $C_{\text {max }}=1$ means that the two systems exactly fulfill the criterion of lag synchronization while unsynchronized systems will result in $C_{\max }$ values close to zero. Note that $C_{\max }$ resembles the similarity function used in [38].

\section{Patient characteristics and data acquisition}

The analyzed EEG signals were recorded from ten epilepsy patients with medically intractable focal epilepsies of the mesial temporal lobe undergoing invasive presurgical diagnostics at the Department of Epileptology of the University of Bonn, Germany. Focal epilepsies are characterized by the fact that seizures do not instantly affect the entire brain but rather begin in a circumscribed region of the brain, called the epileptic focus. Since the localization of the epileptic focus could not be accomplished by means of noninvasive EEG recordings, intracranial electrodes were implanted for the purpose of identifying the focal seizure origin as a prerequisite for possible epilepsy surgery. All patients achieved complete seizure freedom after surgery so the epileptic focus can be assumed to be contained within the resected area.

EEG recordings were performed under video control using stereotactically implanted elastic intrahippocampal depth electrodes (Fig. 1), each equipped with ten cylindrical contacts of a nickel-chromium alloy (length $2.5 \mathrm{~mm}$; intercon- tact distance $4 \mathrm{~mm}$ ). EEG signals were recorded using a 128-channel amplifier system with bandpass filter settings of $0.5-85 \mathrm{~Hz}(12 \mathrm{~dB} /$ octave $)$ using a common average reference. The sampling rate was $173.61 \mathrm{~Hz}$, and analog-digitalconversion was performed at 12-bit resolution.

Data sets for each patient included one or two seizures with a minimum of $10 \mathrm{~min}$ recording time before seizure onset and at least one interictal recording of at least $15 \mathrm{~min}$ length to serve as control. The total number of seizures analyzed in this study was 14. During interictal and preictal recordings, patients were awake and at rest. All recording epochs were free from artifacts. In two of the 14 seizure recordings patients were asked to hyperventilate prior to seizure onset to provoke the onset of the seizure. Epochs were selected by EEG technicians prior to and independently from the design of this study.

Since opinions differ as to how long before a seizure the mechanisms leading to this event actually begin, any recordings within $4 \mathrm{~h}$ prior to seizure onset were excluded from the interictal controls and instead regarded as preictal recordings. In order to neglect the postictal period, which is characterized by an altered appearance of the EEG, recordings within $1 \mathrm{~h}$ after a seizure were discarded from the analysis. Different interictal data sets were usually recorded on different days. The average length of the 35 analyzed interictal EEG recordings was $25 \mathrm{~min}$. The total amount of data analyzed, including seizure recordings, was $31 \mathrm{~h}$. The average interictal recording time per patient was $90 \mathrm{~min}$ (range 15 - $192 \mathrm{~min}$ ). The average preictal recording time was 50 min per seizure (range $10-105 \mathrm{~min}$ ).

\section{Data analysis}

The recorded data were analyzed using a moving-window technique [39]: EEG signals were divided into segments of 4096 sampling points each, corresponding to a window length of $23.6 \mathrm{~s}$ at the given sampling rate, and windows overlapped by $20 \%$ so the distance in time between the starting points of two consecutive windows was $18.9 \mathrm{~s}$. This window length can be regarded as a compromise between the required statistical accuracy for the calculation of the degree of synchronization and approximate stationarity within a window's length $[40,41]$.

Prior to the calculation of the mean phase coherence, three steps of data preprocessing were carried out for each data window. First, the data in each window were demeaned, which corresponds to setting the dc Fourier coefficient ( $\omega$ $=0$ ) to zero. Next, to avoid edge effects, each window was tapered using a cosine half wave (Hanning window) before performing the Fourier transform. Finally, since the calculation of the Hilbert transform in principle requires integration over infinite time, which cannot be performed for a window of finite length, $10 \%$ of the calculated instantaneous phase values were discarded on each end of every window.

Given the symmetry of both synchronization measures, the number of possible combinations of different electrode contacts (ten in each hemisphere) amounts to 190. Taking into account that the statistical significance of the time profiles increases with the mean values of the respective syn- 
chronization measure, which in turn decrease with increasing distance between EEG contacts [22], we restricted the following analysis to neighboring channel combinations, thereby reducing the number of combinations to 18 . Thus, the mean phase coherence $R$ and the maximum linear cross correlation $C_{\max }$ were computed for all combinations of neighboring contacts and for every consecutive window resulting in 18 different time profiles for every recording and for each synchronization measure. Since the algorithm can be implemented employing the fast Fourier transform [42], it is possible to process even a large number of channels in real time on a conventional computer.

\section{E. Detection of a preseizure state}

One aim of this study was to design an automated algorithm to detect a preictal state based on a decrease in phase synchronization described in our earlier work [22] and to distinguish it from the interictal state. In order to obtain a baseline in terms of a reference level for the interictal state, we calculated the mean value $m_{i j}$ and standard deviation $\sigma_{i j}$ for both $R$ and $C_{\max }$ (in the following both denoted by $X$ ) by processing the synchronization profiles from all interictal recordings of a particular patient for every combination of neighboring channels $i$ and $j$.

In relation to any given baseline, a local drop can be characterized by two independent parameters: its depth and duration. The depth of such a drop can be measured in units of the standard deviation of the baseline epoch, whereas its duration can be quantified by the time during which the mean value of a profile drops below a certain threshold.

For a practical implementation, we first applied a backward moving-average filter of width $d$ to smooth the time profiles of $X_{i j}(t)$ and then declared a preictal state if the smoothed profiles $X_{i j}^{d}(t)$ dropped below the interictal mean $m_{i j}$ by more than $r$ standard deviations $\sigma_{i j}$ :

$$
\mathrm{PSD}= \begin{cases}\text { positive } & \text { if } X_{i j}^{d}(t)<m_{i j}-r \sigma_{i j}, \\ \text { negative } & \text { otherwise, }\end{cases}
$$

where PSD stands for preseizure state detection.

Note that this technique is equivalent to using a movingwindow technique, measuring the area between the interictal reference level and the course of the original (unsmoothed) profile within a window of length $d$, and comparing this area to a rectangular reference area of width $d$ and a height of $r$ interictal standard deviations.

Hence, we obtain two parameters $r$ and $d$ that govern the mean depth of a drop over a certain time to be used as a threshold for preictal state detection. In order to estimate both the sensitivity and specificity of the designed algorithm, we applied it to all preictal and all interictal recordings, respectively.

\section{F. Parameter optimization}

In order to determine suitable values for the two parameters $r$ and $d$, we carried out an in-sample optimization for the entire group of patients by maximizing the performance $P$ of our method defined as

$$
P=\sqrt{\frac{f_{\mathrm{se}}^{2}+f_{\mathrm{sp}}^{2}}{2}}
$$

where $f_{\text {se }}$ is the sensitivity, defined as the fraction of seizures for which a preseizure state could be detected, and $f_{\mathrm{sp}}$ is the specificity rate, defined as 1 minus the average number of false positive detections per hour of interictal EEG for the entire group of patients (for more than 1 false positive per hour, $f_{\mathrm{sp}}$ was set to zero for the particular combination of $r$ and $d$ ). For the calculation of the specificity rate, the duration of each interictal recording was reduced by the length $d$ of the moving-average window, keeping it thereby proportional to the specificity (the ratio of true negatives to the number of points in the smoothed profile). Note that there are other ways of defining a performance, e.g., by normalizing the specificity to the duration of the preictal recordings available for each patient, and that they always represent a certain weighting of sensitivity and specificity. For our purpose we have deliberately chosen a performance measure that puts a high emphasis on specificity.

It is important to point out that no a posteriori knowledge in the sense of a "best channel selection" was used, which would require an appropriate statistical correction for multiple testing. For a positive detection at least one of the channel combinations analyzed needed to have a positive PSD and, accordingly, for a negative detection all combinations needed to have negative PSDs. The depth parameter $r$ was varied from 0 to 6 interictal standard deviations while the duration parameter $d$ was varied from 0 to 30 windows (corresponding to $\sim 10 \mathrm{~min}$ ).

\section{G. Cross validation}

As always with in-sample parameter optimization, there is a risk of overtraining an algorithm in the sense that parameters are optimized on a given sample to yield a performance for this sample (estimated performance) that may not be achieved in an out-of-sample trial (true performance). To reduce the risk of an overestimation of our method's performance due to in-sample optimization, we performed a cross validation analysis. For the given sample of patients, a tenfold cross validation was carried out using the "leaving-oneout" method $[43,44]$, i.e., each of the ten patients was subsequently used as a test sample while the remaining nine patients served as training samples, i.e., for parameter optimization. The overall performance of the individual test samples was then compared to the performance of the entire group as defined in the previous section. Similar values for both performance estimates indicate that the parameters have not been overtrained. The standard error of the sensitivity is given by

$$
E=\sqrt{\frac{f_{\mathrm{se}}\left(1-f_{\mathrm{se}}\right)}{n}}
$$

where $n$ is the number of test cases (i.e., $n=10$ in this study). The standard error for the specificity is calculated accordingly, and the resulting error of the performance can be calculated using Gaussian error propagation. 
interictal $\left(R=0.83 ; C_{\max }=0.91\right)$

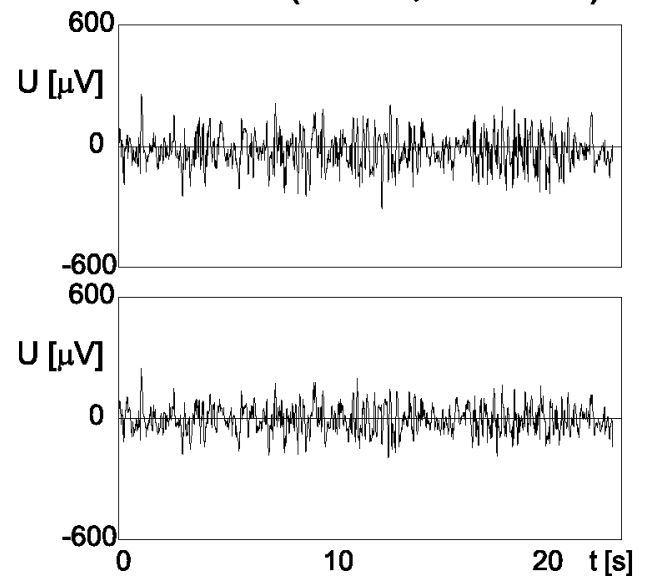

preictal $\left(R=0.37 ; C_{\max }=0.48\right)$
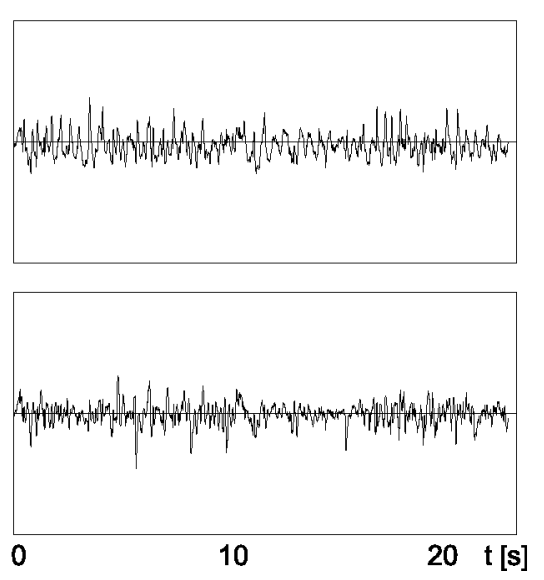

ictal $\left(R=0.94 ; C_{\max }=0.92\right)$
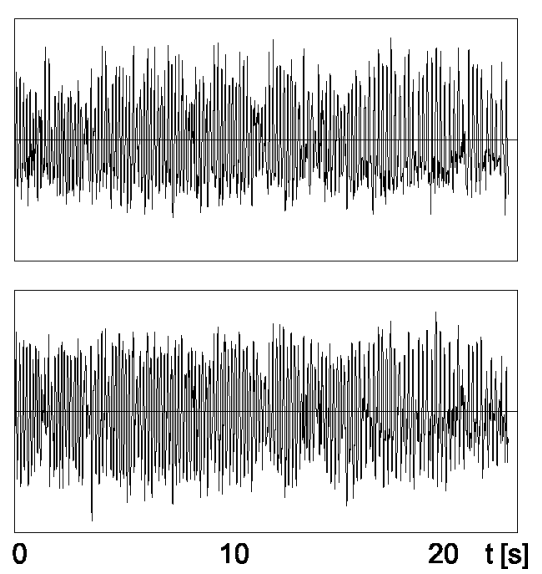

FIG. 2. Examples of EEG segments from neighboring channels of one patient during the interictal, preictal, and ictal state as well as values for the mean phase coherence $R$ and the maximum linear cross correlation $C_{\max }$ calculated from these segments.

\section{H. Test for statistical validity}

Time series such as the profiles $X_{i j}(t)$ that were obtained from measured data usually contain fluctuations and therefore in principle have a nonzero probability of crossing any threshold within their range of definition. This has to be kept in mind whenever a threshold criterion is applied to real data. Concerning our analysis, this means that there is no guarantee that the observed effects are indeed due to different characteristics of the interictal and preictal state but instead could be caused, at least in part, by these fluctuations. Given the respective duration of the interictal and preictal recordings, there is a nonzero probability of finding a "preictal" drop in at least one of the 18 possible combinations of neighboring channels just by chance. Therefore, the null hypothesis to be tested is that the observed performance values are merely caused by fluctuations in synchronization that are not related to the existence of a preictal state.

With this aim, we designed a surrogate of the analyzed set of recordings [45]. For each patient and every recording site we replaced any preictal interval (i.e., any recording within 4 $\mathrm{h}$ before seizure onset) by randomly selected interictal ones and vice versa, applying constraints to keep the ratio of preictal and interictal time constant for the entire group and to maximize the overall amount of data contained in the surrogate set. The same process of preictal state detection and optimization was then applied to this surrogate set of recordings. To test the null hypothesis, the maximum performance found for the original set of recordings was compared to that for the surrogate set.

\section{RESULTS}

Examples of the raw data analyzed in this study are given in Fig. 2. Displayed are original EEG segments recorded simultaneously from two neighboring recording sites of a patient during the seizure-free interval, during the period preceding a seizure, and during the seizure itself, along with the corresponding values for each of the two synchronization measures $R$ and $C_{\text {max }}$.

Figure 3 shows an illustration of the detection technique described above for an interictal EEG recording and a recording containing a seizure from one patient. It is easy to note that preictal synchronization values are lower than interictal values. A remarkable aspect is the similarity of the profiles for the two different measures for synchronization. To quantify this similarity the linear correlation coefficient $r^{2}$ between the two synchronization measures was calculated for all possible channel combinations for all profiles of each patient. Correlation coefficients were found to range from 0.32 to 0.69 for different patients, indicating a medium degree of linear correlation between the two measures.

The dependence of the performance of the algorithm on the parameters $r$ and $d$ in terms of both sensitivity and specificity is illustrated by Fig. 4. Optimum performance for the mean phase coherence $R$ could be obtained, e.g., for values $r=4$ and $d \approx 2.5 \mathrm{~min}$ (eight windows), and amounted to $P$ $=0.93$ for the entire group. For the maximum linear cross correlation $C_{\text {max }}$, parameter values of, e.g., $r=3.8$ and $d$ $\approx 3.5$ min (11 windows) led to the same maximum performance. Using these parameters for the ten patients analyzed in this study, we found positive PSDs in 12 out of 14 seizure recordings corresponding to eight out of ten patients. For the given parameters there was not a single false positive detection during the entire $15 \mathrm{~h}$ of interictal recordings for either measure, corresponding to a specificity of 1 for the whole group of patients. Concerning the variability of optimum parameters among the different patients, we found that for each of the eight patients for whom a preseizure state could be detected, there was a large contiguous region of parameter combinations rendering a performance of 1 , while for the remaining patients there was no such region. These two patients were the same for both synchronization measures.

The number of channel combinations with positive PSD for the remaining patients ranged from 1 to 7 (mean 3 ) for $R$ and from 1 to 8 (mean 3 ) for $C_{\max }$ for the different seizures. For all but one seizure with positive PSD, there was at least one channel combination exhibiting positive PSD for both measures. Furthermore, this combination was usually the one that exhibited the most prominent drops in synchronization for both measures. 
interictal

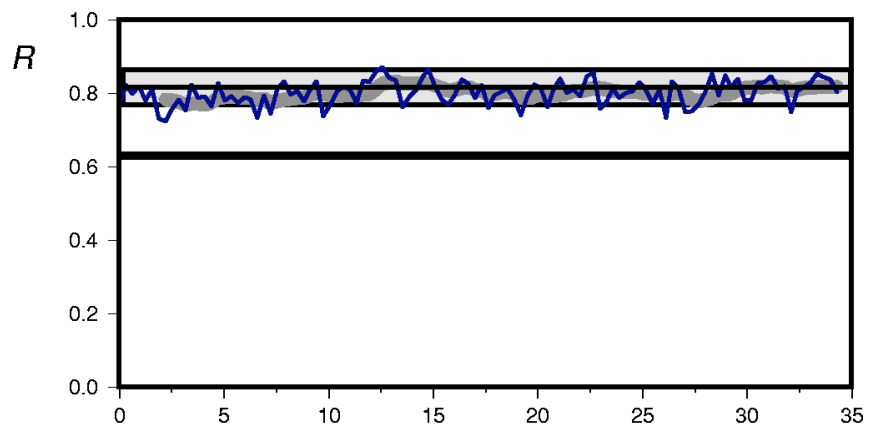

preictal

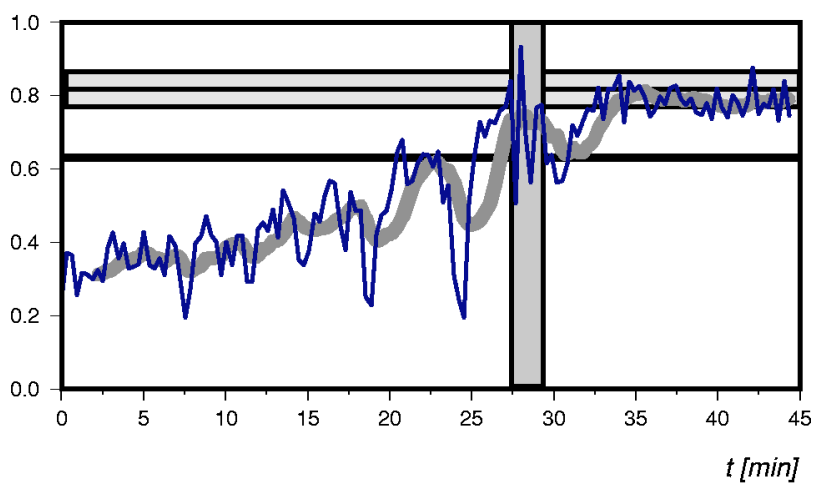

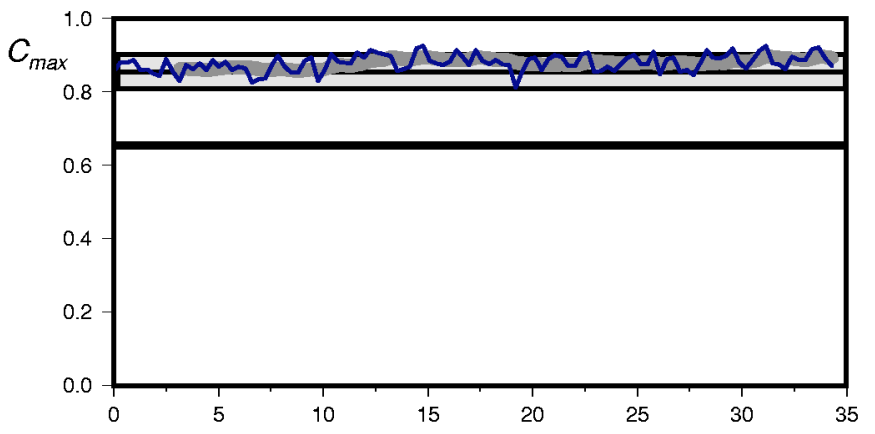

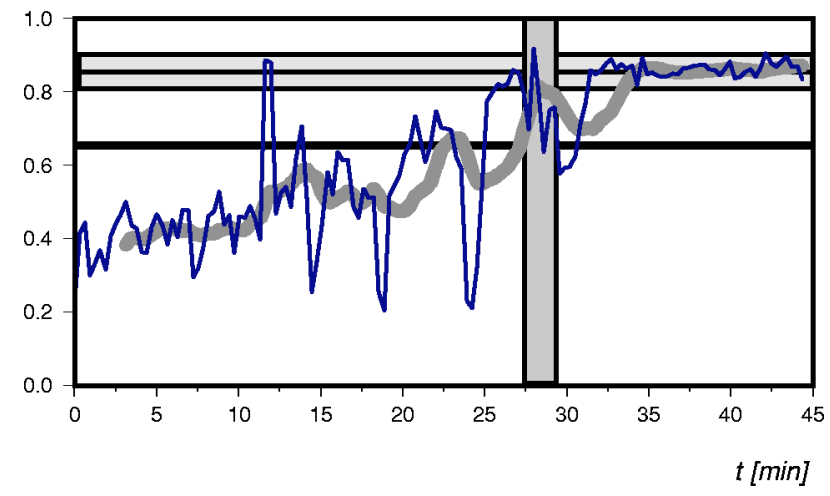

FIG. 3. (Color online) Interictal and preictal synchronization profiles from one patient. Displayed are both the original and the smoothed profiles of one channel combination for both the mean phase coherence $R$ (upper row) and the maximum linear cross correlation $C_{\text {max }}$ (lower row) along with the mean value and standard deviation (horizontal gray bars) calculated from the interictal recordings as well as the detection threshold (horizontal thick black line) calculated from these values using optimized parameters. The seizure is marked by a gray vertical bar. Note that the preictal state has already started at the beginning of the recording containing the seizure.

The duration of the detected preictal state could not exactly be determined in most cases since the preictal state had already started at the beginning of the preictal recordings. The anticipation time for a seizure was defined as the time interval between the first positive PSD found in any of the combinations of neighboring channels during the preictal period of $4 \mathrm{~h}$ before the seizure and the seizure onset and ranged, as determined from the data available, from 4 min up to more than 219 min (mean $86 \mathrm{~min}$ ) for the mean phase coherence and from 7 min up to more than 218 min (mean $102 \mathrm{~min}$ ) for the maximum linear cross correlation. Anticipation times exceeding the preictal recording time are due to gaps in these recordings. The difference in anticipation times found for the two measures did not exceed 10 min except for two seizures where anticipation times for $C_{\max }$ were significantly longer than for $R$.

Analyses of the preictal drops revealed that in 9 out of the 12 detected preictal states, at least for one combination the preictal drop lasted until the onset of the seizure whereas in the remaining cases the preictal synchronization level rose above the PSD threshold before (although usually very close to) seizure onset. During the actual seizure activity, the usual finding was a steep increase in synchronization reaching or even surpassing the interictal mean value. As for the postictal period, we found that in 7 out of the 12 seizures with positive
PSD, the degree of synchronization remained above the PSD threshold while in the remaining cases it started decreasing shortly after the seizure.

Results of the cross validation yielded a corrected performance of $P=0.91$ for the mean phase coherence that did not deviate from the in-sample performance given above by more than the standard error of $E=0.11$. For the maximum linear cross correlation, the corrected performance was exactly the same as the original performance.

As a check for statistical validity of the observed effects, the surrogate test was carried out as described above. Results of the parameter optimization for the surrogate set of recordings are displayed in Fig. 5. Note that for both synchronization measures the maximum the performance is obtained for parameters yielding either a high sensitivity or a high specificity, but no parameter values could be found for which both sensitivity and specificity rate simultaneously exceed a value of 0.54 .

\section{DISCUSSION}

\section{A. Preseizure state detection}

Results show that the period preceding a seizure can be characterized by a decrease in synchronization between dif- 
Mean Phase Coherence $\boldsymbol{R}$
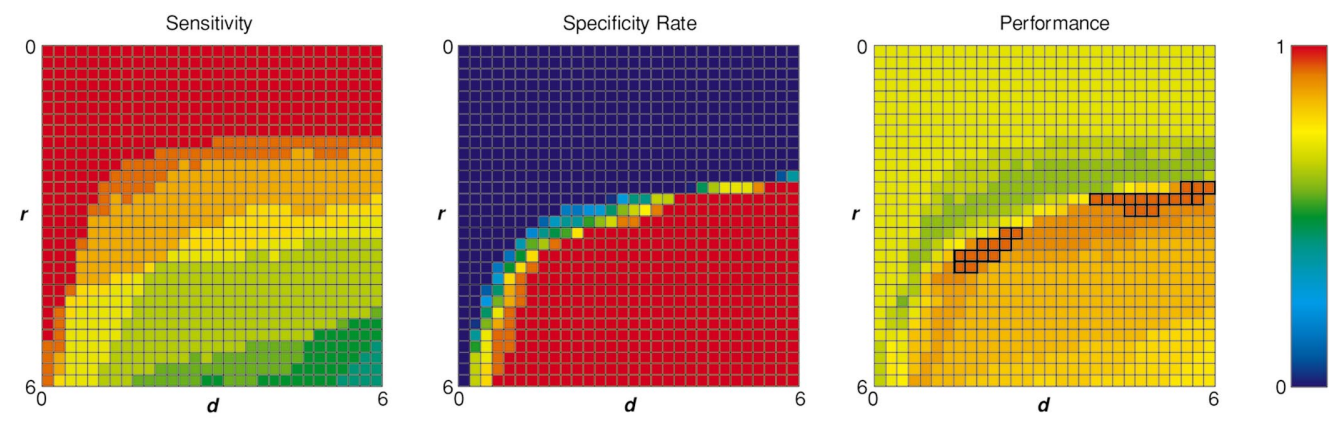

Maximum Linear Cross Correlation $\boldsymbol{C}_{\max }$
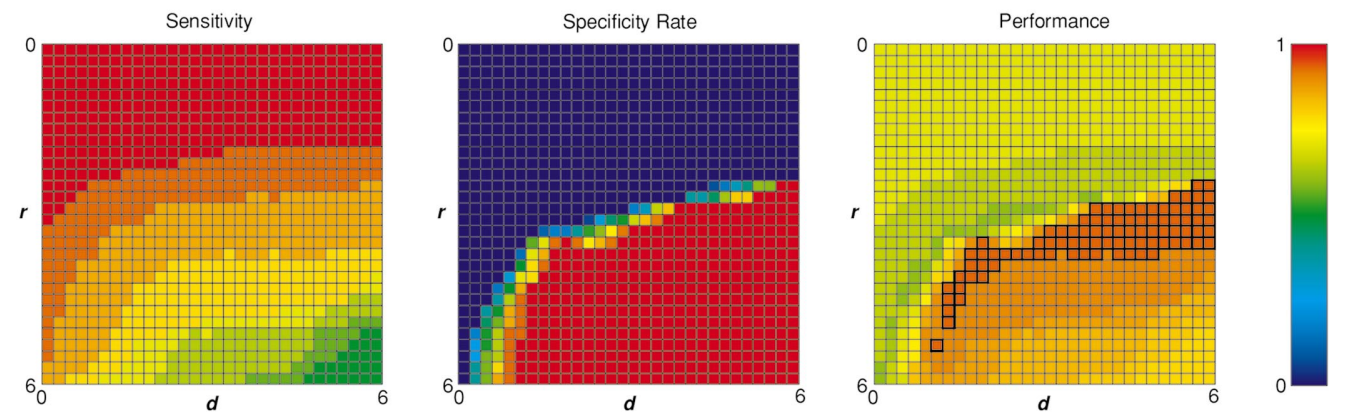

FIG. 4. (Color) Optimization of parameters (see text). Color-coded values for sensitivity, specificity rate, and performance are displayed in dependence on the parameters $d$ (filter width in minutes) and $r$ (average depth of drops in units of the interictal standard deviation) for both $R$ (upper row) and $C_{\max }$ (lower row). Parameter combinations yielding maximum performance are marked by bold frames.

Mean Phase Coherence $\boldsymbol{R}$
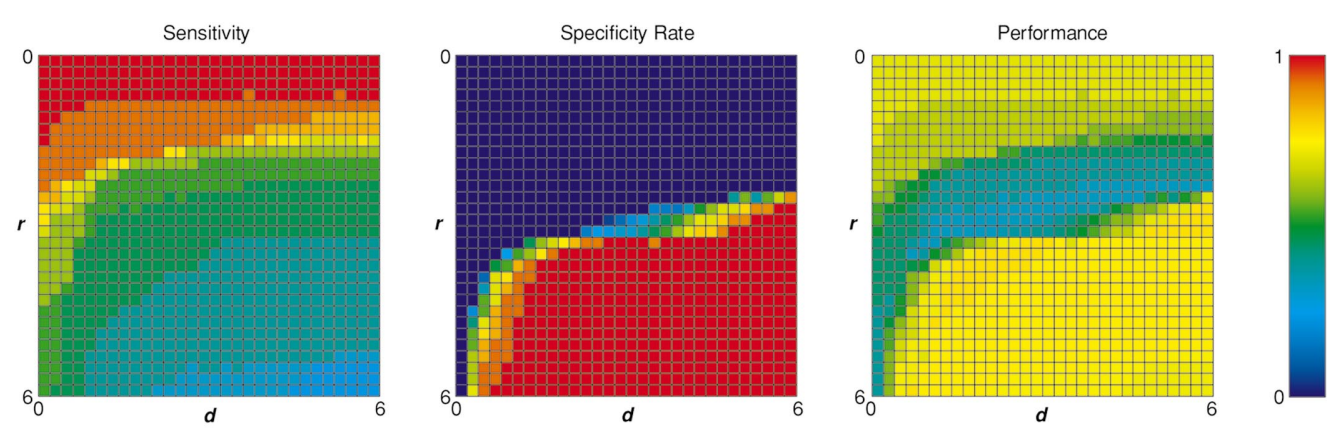

Maximum Linear Cross Correlation $\boldsymbol{C}_{\max }$
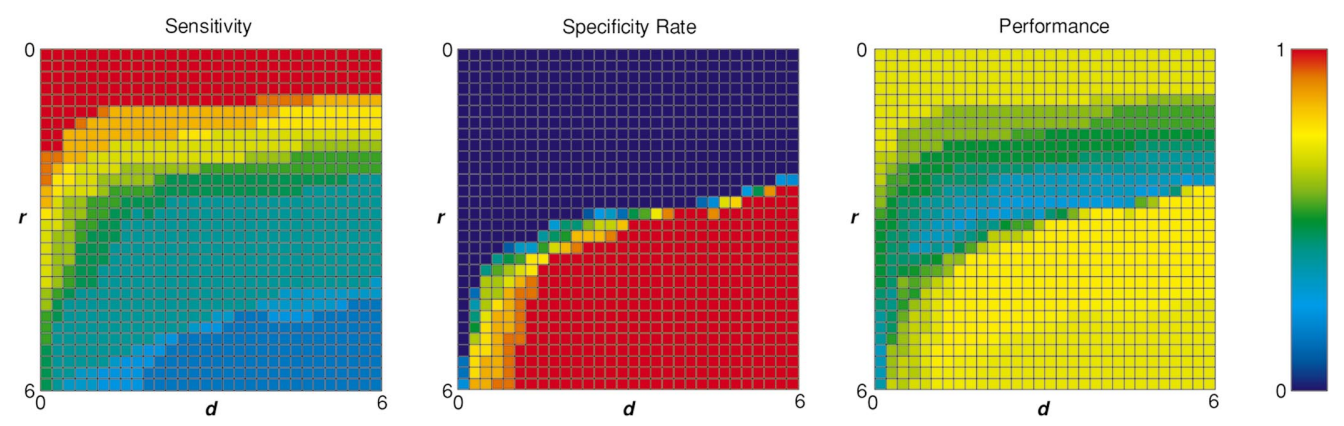

FIG. 5. (Color) Same as Fig. 4, but for the surrogate set of recordings. 
ferent EEG recording sites. This decrease in synchronization can be detected using the automated technique introduced in this study. The overall performance of the method based on the given data indicates that it works well for most patients although it fails in some cases. The optimization of the parameters used in the detection algorithm was carried out for the entire group of patients and can be regarded as for the most part free from overtraining as confirmed by cross validation. (To actually prove this point, validation in an out-ofsample trial would be necessary, which, however, goes beyond the scope of this study.) The results of the surrogate test for statistical validity indicate that our findings are indeed caused by different characteristics of the interictal and preictal periods and cannot be explained by merely random fluctuations of the synchronization profiles.

\section{B. Comparison of the synchronization measures}

When comparing the mean phase coherence as a measure for phase synchronization and the maximum linear cross correlation as a measure for lag synchronization it should be kept in mind that the latter depends on both amplitudes and phases of the time series while the former depends solely on the phases, i.e., in the case of phase synchronization amplitudes may be completely uncorrelated or even chaotic.

As for the duration of the detected state it is possible to distinguish between the anticipation horizon (the time between detection and seizure onset) and the actual duration of the preictal state (from the beginning of a significant drop to seizure onset) where the difference between the two is given by the above defined parameter $d$. For both of the different synchronization measures, the duration of the anticipation times is mostly of the order of several tens of minutes. This stands in contrast to earlier studies [11-17] where mean anticipation times from 2 to $11 \mathrm{~min}$ have been reported. The different range of anticipation times implies that changes in dynamics tracked by synchronization measures are different from those tracked by other, mostly univariate nonlinear measures. It should not be omitted that in some recent studies $[18,19,21]$ similar anticipation times have been reported as found in this study. Whether these findings reflect the same dynamical aspects, remains to be investigated. It should be pointed out that with the extended anticipation horizons found in this study, there is little information about when exactly the seizure will occur. We therefore avoid the term "prediction" and rather speak of "anticipation" of seizures, implying that we may know that a seizure has a certain probability to occur within a certain time frame but we do not know exactly when. An anticipation horizon of this length could on the other hand enable patients to respond to the threat of an impending seizure.

Concerning the location of the observed preictal drops in synchronization, it turned out that for half of the patients with positive PSDs (four out of eight), the most prominent preictal drops in synchronization were found in the nonfocal hemisphere, while for the remaining patients they were found in the focal hemisphere, although not necessarily within the focus (as determined by electrographical seizure onset) itself. This effect was found for both synchronization measures and stands in agreement with earlier studies (e.g., $[15,19])$ while in other studies seizure precursors were mostly found within or near the focal area (e.g., $[11,18]$ ). This finding indicates that the process of seizure generation in focal epilepsy may not necessarily be confined to the focal area but may instead involve more distant areas of brain tissue, even in the nonfocal hemisphere.

Evaluation of the postictal period shows that in the majority of cases the interictal level is retained after the seizure regardless of which measure was evaluated. This effect corresponds well to the hypothesis of a seizure acting as a resetting mechanism from unstable dynamics to a more stable one [46]. Such a resetting phenomenon, however, was not found in all cases, which could be interpreted in the sense that the resetting attempt is not always successful, particularly as there was no apparent correlation of a "futile resetting" with an increased occurrence of subsequent seizures.

The results of this study generally show a similar performance for both synchronization measures. In particular, the outcome of the patients in terms of positive or negative PSD was the same for both measures. Furthermore, anticipation times were found to be in the same range, and combinations with positive PSD matched for both measures in the majority of patients. This indicates that the preictal changes in dynamics analyzed in this study can be sufficiently characterized using a linear measure. If these changes in synchronization had been nonlinear in nature, we would have expected a superior performance of the mean phase coherence as a nonlinear measure. A way to further investigate this issue would be the use of bivariate surrogates [47] to discern linear aspects of synchronization from nonlinear ones which, however, goes beyond the scope of this study.

\section{Future perspectives}

For the four patients with more than one seizure with positive PSD, we found that although the PSD was usually restricted to a few channel combinations, in three cases there was at least one combination for which the PSD was positive for all seizures of a particular patient. Also, it was usually this combination that exhibited the most prominent drops in synchronization. This finding stands in contrast to earlier studies $[14,19]$ where electrode sites exhibiting predictive features were reported to change from seizure to seizure for the same patient. The described stability over seizures could be used to further improve the specificity of the method by only considering channel combinations for a particular patient that have proven to be sensitive for preictal state detection, and thus omitting possible false positive detections in the remaining channel combinations. (As expected for patients analyzed in this study, such a "best channel selection" yielded an even better performance but also a lower statistical validity as determined by the corresponding surrogate test.) Another way to improve the method's performance in long-term evaluations could be an individual parameter adjustment for each patient to obtain the best possible discrimination between interictal and preictal states. It should be pointed out, however, that both of these improvements would 
require a great deal of preanalysis and additional training data for each patient in order to obtain the necessary information.

In this context it is important to once again emphasize that the results described above were obtained from a retrospective study on selected data samples in the sense that, for instance, any sleep recordings were excluded from the set of interictal data. The sensitivity and specificity obtained in this study might therefore significantly differ from what one would find in a prospective study. The aim of this study was merely to examine whether a statistically valid discrimination between preictal states and selected interictal states is in principle possible with the method introduced. From our preliminary experience with long-term recordings we would conjecture that including unselected interictal data such as different states of vigilance could decrease the predictive performance, whereas best channel selection and patientspecific parameters will on the other hand be likely to result in an increase in performance. The extended anticipation horizons found in this study would leave enough time for intervention strategies such as automated on-demand medication [48,49], electrical stimulation [50-52], or local cooling [53], provided that a sufficient performance of the method introduced can be obtained in a prospective setting.

In order to adapt this method to such a prospective study design for continuous recordings, it is necessary to realize that the definition of a baseline becomes a nontrivial problem. To avoid false detections due to trends or shifts in baseline we would recommend the use of an adaptive baseline. A simple realization of such an adaptive baseline could, for instance, be given by defining the adaptive mean $m_{t}$ as

$$
m_{t}=\frac{w}{T} \sum_{k=-T / 2 w}^{T / 2 w} X_{t+k-L / w}
$$

where $t$ is the time index of the continuous time series of the measure $X_{t}=X(t), w$ is the time interval between two consecutive analysis windows (i.e., two consecutive values $X_{t}$ ), $T$ is the duration of the baseline epoch, and $L$ is the time lag between the (moving) baseline and the time of (prospective) analysis. The standard deviation for the adaptive baseline should, of course, be determined accordingly. From our preliminary experience with long-term recordings, we would propose to use, for instance, a time lag of $L=24 \mathrm{~h}$ to adapt to circadian fluctuations and a baseline length $T$ of a few hours.

\section{CONCLUSION}

Based on the findings of a preictal loss in synchronization between EEG signals recorded simultaneously from different locations in the brain, we have investigated the suitability of this phenomenon for characterization of the preseizure state. By evaluating both sensitivity and specificity, by checking for in-sample overtraining via cross validation, and by applying a surrogate test for statistical validity, we have demonstrated that our method for preictal state detection in principle allows us to discriminate the preictal from the interictal state, thus satisfying a necessary condition for prospective seizure anticipation. The similar performance of the two different measures for synchronization, one linear and one nonlinear, indicates that the changes in dynamics before seizures that were found in this study are not necessarily caused by nonlinear features of the dynamics.

\section{ACKNOWLEDGMENTS}

We are grateful to Martin Kurthen for useful discussion and valuable comments. This work was supported by the Deutsche Forschungsgemeinschaft.
[1] S. S. Viglione and G. O. Walsh, Electroencephalogr. Clin. Neurophysiol. 39, 435 (1975).

[2] Z. Rogowski, I. Gath, and E. Bental, Biol. Cybern. 42, 9 (1981).

[3] R. B. Duckrow and S. S. Spencer, Electroencephalogr. Clin. Neurophysiol. 82, 415 (1992).

[4] J. Gotman, J. Ives, P. Gloor, A. Olivier, and L. Quesney, Epilepsia 23, 432 (1982).

[5] H. H. Lange, J. P. Lieb, J. Engel, Jr., and P. H. Crandall, Electroencephalogr. Clin. Neurophysiol. 56, 543 (1983).

[6] H. G. Wieser, Epilepsia 30, 669 (1989).

[7] A. Katz, D. A. Marks, G. McCarthy, and S. S. Spencer, Electroencephalogr. Clin. Neurophysiol. 79, 153 (1991).

[8] L. D. Iasemidis, J. C. Sackellares, H. P. Zaveri, and W. J. Williams, Brain Topogr. 2, 187 (1990).

[9] L. D. Iasemidis, L. D. Olson, R. S. Savit, and J. C. Sackellares, Epilepsy Res. 17, 81 (1994).

[10] K. Lehnertz and C. E. Elger, Electroencephalogr. Clin. Neurophysiol. 95, 108 (1995).

[11] K. Lehnertz and C. E. Elger, Phys. Rev. Lett. 80, 5019 (1998).

[12] C. Elger and K. Lehnertz, Eur. J. Neurosci. 10, 786 (1998).
[13] J. Martinerie, C. Adam, M. Le Van Quyen, M. Baulac, S. Clemenceau, B. Renault, and F. J. Varela, Nat. Med. 4, 1173 (1998).

[14] M. Le Van Quyen, J. Martinerie, M. Baulac, and F. Varela, NeuroReport 10, 2149 (1999).

[15] M. Le Van Quyen, C. Adam, J. Martinerie, M. Baulac, S. Clémenceau, and F. Varela, Eur. J. Neurosci. 12, 2124 (2000).

[16] M. Le Van Quyen, J. Martinerie, V. Navarro, P. Boon, M. D’Havé, C. Adam, B. Renault, M. Baulac, and F. Varela, Lancet 357, 183 (2001).

[17] V. Navarro, J. Martinerie, M. Le Van Quyen, S. Clemenceau, C. Adam, M. Baulac, and F. Varela, Brain 125, 640 (2002).

[18] B. Litt et al., Neuron 30, 183 (2001).

[19] L. D. Iasemidis, P. Pardalos, J. C. Sackellares, and D. S. Shiau, J. Combinatorial Optimization 5, 9 (2001).

[20] J. Arnhold, P. Grassberger, K. Lehnertz, and C. E. Elger, Physica D 134, 419 (1999).

[21] K. Schindler, R. Wiest, M. Kollar, and F. Donati, Clin. Neurophysiol. 113, 604 (2002).

[22] F. Mormann, K. Lehnertz, P. David, and C. E. Elger, Physica D 144, 358 (2000). 
[23] M. Le Van Quyen, J. Martinerie, V. Navarro, M. Baulac, and F. J. Varela, J. Clin. Neurophysiol. 95, 108 (2001).

[24] C. Huygens, Horologium Oscillatorum (Apud F. Muguet, Paris, 1673).

[25] A. Pikovsky, M. Rosenblum, and J. Kurths, Synchronization: A Universal Concept in Nonlinear Sciences (Cambridge University Press, Cambridge, U.K., 2001).

[26] M. G. Rosenblum, A. S. Pikovsky, and J. Kurths, Phys. Rev. Lett. 76, 1804 (1996).

[27] C. Schaefer, M. G. Rosenblum, J. Kurths, and H. H. Abel, Nature (London) 392, 239 (1998).

[28] M. G. Rosenblum, J. Kurths, A. S. Pikovsky, C. Schaefer, P. Tass, and H. H. Abel, IEEE Eng. Med. Biol. Mag. 17, 46 (1998).

[29] C. Schaefer, M. G. Rosenblum, H. H. Abel, and J. Kurths, Phys. Rev. E 60, 857 (1999).

[30] R. Mrowka, A. Patzak, and M. Rosenblum, Int. J. Bifurcation Chaos Appl. Sci. Eng. 10, 2479 (2000).

[31] P. Tass, M. G. Rosenblum, J. Weule, J. Kurths, A. Pikovsky, J. Volkmann, A. Schnitzler, and H. J. Freund, Phys. Rev. Lett. 81, 3291 (1998).

[32] R. Quian Quiroga, A. Kraskov, T. Kreuz, and P. Grassberger, Phys. Rev. E 65, 041903 (2002).

[33] K. V. Mardia, Probability and Mathematical Statistics: Statistics of Directional Data (Academic Press, London, 1972).

[34] M. Rosenblum, A. S. Pikovsky, J. Kurths, C. Schaefer, and P. A. Tass, in Handbook of Biological Physics, edited by F. Moss and S. Gielen (Elsevier Science, Amsterdam, 2001), pp. 297-321.

[35] D. Gabor, Proc. IEEE 93, 429 (1946).

[36] P. Panter, Modulation, Noise, and Spectral Analysis (McGrawHill, New York, 1965).

[37] J. P. Lachaux, E. Rodriguez, J. Martinerie, and F. J. Varela, Hum. Brain Mapp. 8, 194 (1999).

[38] M. G. Rosenblum, A. S. Pikovsky, and J. Kurths, Phys. Rev. Lett. 78, 4193 (1997).
[39] J. S. Barlow, J. Clin. Neurophysiol. 2, 267 (1985).

[40] F. H. Lopes da Silva, in Electroencephalography, Basic Principles, Clinical Applications and Related Fields, edited by E. Niedermayer and F. H. Lopes da Silva (Urban and Schwarzenberg, Baltimore, MD, 1987), pp. 871-897.

[41] S. Blanco, H. Garcia, R. Quian Quiroga, L. Romanelli, and O. A. Rosso, IEEE Eng. Med. Biol. Mag. 4, 395 (1995).

[42] W. H. Press, B. Flannery, S. Teukolsky, and W. Vetterling, Numerical Recipes in Pascal: The Art of Scientific Computing (Cambridge University Press, Cambridge, U.K., 1989).

[43] V. N. Vapnik, Estimation of Dependence Based on Empirical Data (Springer, New York, 1982).

[44] V. S. Cherkassky and F. M. Mulier, Learning from Data: Concepts, Theory, and Methods (John Wiley and Sons, New York, 1998).

[45] R. G. Andrzejak, F. Mormann, T. Kreuz, C. Rieke, C. E. Elger, and K. Lehnertz, Phys Rev. E 67, 010901 (2003).

[46] J. C. Sackellares, L. D. Iasemidis, P. M. Pardalos, and D.-S. Shiau, in Biocomputing, edited by P. M. Pardalos and J. Principe (Kluwer Academic Publishers, Dordrecht, 2002).

[47] T. Schreiber and A. Schmitz, Physica D 142, 346 (2000).

[48] H. G. Eder, D. B. Jones, and R. S. Fisher, Epilepsia 38, 516 (1997).

[49] A. G. Stein, H. G. Eder, D. E. Blum, A. Drachev, and R. S. Fisher, Epilepsy Res. 39, 103 (2000).

[50] S. J. Schiff, K. Jerger, D. H. Duong, T. Chang, M. L. Spano, and W. L. Ditto, Nature (London) 370, 615 (1994).

[51] M. Velasco, F. Velasco, A. L. Velasco, B. Boleaga, F. Jimenez, F. Brito, and I. Marquez, Epilepsia 41, 158 (2000).

[52] B. J. Gluckman, H. Nguyen, S. L. Weinstein, and S. J. Schiff, J. Neurosci. 21, 590 (2001).

[53] M. W. Hill, M. Wong, A. Amarakone, and S. M. Rothman, Epilepsia 41, 1241 (2000).

[54] F. Mormann, T. Kreuz, R. G. Andrzejak, P. David, K. Lehnertz, and C. E. Elger, Epilepsy Res. (to be published). 